\title{
STUDENT PROCESSES
}

\author{
C. C. HEYDE, ${ }^{*}$ Australian National University and Columbia University \\ N. N. LEONENKO,** Cardiff University
}

\begin{abstract}
Stochastic processes with Student marginals and various types of dependence structure, allowing for both short- and long-range dependence, are discussed in this paper. A particular motivation is the modelling of risky asset time series.

Keywords: Student distribution; Ornstein-Uhlenbeck-type process; self-decomposability; long-range dependence
\end{abstract}

2000 Mathematics Subject Classification: Primary 60E99; 60G10; 60G35; 62M10

\section{Introduction}

It is now generally accepted that heavy-tailed distributions occur commonly in practice. Their use is now widespread in communications network, risky asset, and insurance modelling. However, the study of stationary processes having these heavy-tailed distributions as their one-dimensional distributions, and also having a full range of possible dependence structures, has received rather little attention. In this paper, we focus on such processes with Student $t$ marginals. The Student $t$ family with $v$ degrees of freedom covers the range of power tail possibilities, the spectrum including the Cauchy distribution $(v=1)$ and ranging through to the Gaussian distribution as $v \rightarrow \infty$.

In the field of finance, distributions of logarithmic asset returns can often be fitted extremely well by Student $t$-distributions (see, for instance, Hurst et al. (1997), Hurst and Platen (1997), Heyde (1999), Heyde and Liu (2001), Heyde and Gay (2002), and Bingham and Kiesel (2002)). All these authors have advocated using a $t$-distribution with $v$ degrees of freedom, typically such that $3 \leq v \leq 5$. This implies infinite $k$ th moments, for $k \geq v$.

Another issue in modelling economic and financial time series is that their sample autocorrelation functions may decay quickly, but their absolute or squared increments may have autocorrelation functions with nonnegligible values for large lags (Heyde and Yang (1997), Heyde (1999), Heyde and Liu (2001), Anh et al. (2002)). These ubiquitous phenomena call for an effort to develop reasonable models that can be integrated into economic and financial theory as well as theories of turbulence (see, for instance, Woyczyński (1998)). This approach has a long history, certainly dating back to Mandelbrot's work in the 1960s (see Mandelbrot (2001a), (2001b) and references therein), in which the use of (stable or Pareto-type) heavy-tailed distributions was advocated.

Alternatively, Barndorff-Nielsen (1998), Barndorff-Nielsen et al. (1998), Barndorff-Nielsen and Pérez-Abreu (1999), Barndorff-Nielsen and Shephard (2001), and Barndorff-Nielsen et al.

Received 16 September 2004; revision received 15 February 2005.

* Postal address: Mathematical Sciences Institute, Australian National University, Canberra, ACT 0200, Australia. Email address: chris@maths.anu.edu.au

** Postal address: Cardiff School of Mathematics, Cardiff University, Senghennydd Road, Cardiff CF24 4AG, UK. Email address: leonenkon@ cardiff.ac.uk 
(2002) (see also Madan and Seneta (1990), Eberlein and Keller (1995), Bibby and Sørensen (1997), Sørensen and Bibby (2003), and Seneta (2004)) have proposed the use of hyperbolic distributions in financial econometrics. In fact, the symmetric scaled $t$-distribution can be considered to be a limiting case of the generalized hyperbolic distribution (see BarndorffNielsen (1977), (1978), Barndorff-Nielsen and Pérez-Abreu (1999), Barndorff-Nielsen and Shephard (2001), and Sørensen and Bibby (2003) for details). However, some properties of the $t$-distribution cannot be obtained from the corresponding limiting procedure. For instance, the characteristic function of the symmetric scaled $t$-distribution cannot be obtained from the expression for the characteristic function of the generalized hyperbolic distribution, as this is based on the Laplace transform technique, which does not exist for the symmetric scaled $t$-distribution (see Remark 2.3, below). Moreover, most of the hyperbolic distributions are semiheavy tailed, while the symmetric scaled $t$-distribution is heavy tailed.

In this paper, we propose a number of stochastic processes with Student marginals and various types of dependence structure that are, in the authors' opinion, relevant for economics and finance. Our emphasis is on processes with dependent increments, but it has been known since the paper of Grosswald (1976) that the $t$-distribution is infinitely divisible; Student processes (with independent increments) therefore exist as Lévy processes. Financial applications in this context have recently been widely discussed in the literature (see, for instance, Cont and Tankov (2004) and Schoutens (2003)).

We shall use the following standard notation for characteristic functions, cumulant transforms, and Laplace transforms, respectively, of a random variable $X$ :

$$
\begin{aligned}
\phi_{X}(\zeta) & =\mathrm{E}\left\{\mathrm{e}^{\mathrm{i} \zeta X}\right\}, \\
\kappa_{X}(\zeta) & =\log \mathrm{E}\left\{\mathrm{e}^{\mathrm{i} \zeta X}\right\}, \\
L T_{X}(\zeta) & =\mathrm{E}\left\{\mathrm{e}^{-\zeta X}\right\} .
\end{aligned}
$$

We shall also use the notation $X \stackrel{\mathrm{D}}{=} Y$ for equality of distributions of two random variables $X$ and $Y$, or $X_{t} \stackrel{\mathrm{D}}{=} Y_{t}$ for equality of the finite-dimensional distributions of stochastic processes.

\section{The symmetric scaled $t$-distribution and Student Lévy processes}

In this section, we summarize some (mostly known) results from $t$-distribution theory that will be needed below.

\subsection{Density and characteristic functions}

The symmetric scaled $t$-distribution $T(\nu, \delta, \mu)$ with $v>0$ degrees of freedom can be defined by the probability density function (PDF)

$$
\operatorname{student}(x)=c(v, \delta) \frac{1}{\left[1+((x-\mu) / \delta)^{2}\right]^{(v+1) / 2}}, \quad x \in \mathbb{R},
$$

where $\delta>0$ is a scaling parameter, $\mu \in \mathbb{R}$ is a location parameter, and

$$
c(v, \delta)=\frac{\Gamma\left(\frac{1}{2}(v+1)\right)}{\delta \sqrt{\pi} \Gamma\left(\frac{1}{2} v\right)} .
$$

Remark 2.1. Note that a random variable $X \sim T(\nu, \delta, \mu)$ has the representation

$$
X \stackrel{\mathrm{D}}{=} \mu+\sigma \varepsilon
$$


where the independent random variables $\varepsilon$ and $\sigma^{2}$ have the standard normal distribution $N(0,1)$ and the inverse (reciprocal) gamma distribution $\mathrm{R} \Gamma\left(\frac{1}{2} \nu, \frac{1}{2} \delta^{2}\right)$, respectively.

Recall that a random variable $\gamma_{\beta, \alpha}$ has a gamma distribution $\Gamma(\beta, \alpha), \beta, \alpha>0$, if its PDF takes the form

$$
\operatorname{gamma}(x)=\frac{\alpha^{\beta}}{\Gamma(\beta)} x^{\beta-1} \mathrm{e}^{-\alpha x}, \quad x>0,
$$

and a random variable $r_{\beta, \alpha}=1 / \gamma_{\beta, \alpha}$ has an inverse gamma distribution $\mathrm{R} \Gamma(\beta, \alpha)$ if its $\mathrm{PDF}$ is of the form

$$
\operatorname{invgamma}(x)=\frac{\alpha^{\beta}}{\Gamma(\beta)} x^{-\beta-1} \mathrm{e}^{-\alpha / x}, \quad x>0 .
$$

Note that $\gamma_{\beta, \alpha} \stackrel{\mathrm{D}}{=} \gamma_{\beta, 1} / \alpha, r_{\beta, \alpha} \stackrel{\mathrm{D}}{=} \alpha r_{\beta, 1}, \mathrm{E}\left\{\gamma_{\beta, \alpha}\right\}=\beta / \alpha$, and $\mathrm{E}\left\{\gamma_{\beta, \alpha}^{2}\right\}=\beta(\beta+1) / \alpha^{2}$. Only moments less than $\beta$ exist for $\mathrm{R} \Gamma(\beta, \alpha)$, with $\mathrm{E}\left\{r_{\beta, \alpha}\right\}=\alpha /(\beta-1), \beta>1$, and $\operatorname{var}\left\{r_{\beta, \alpha}\right\}=\alpha^{2} /\left[(\beta-1)^{2}(\beta-2)\right], \beta>2$.

Both PDFs (2.3) and (2.4) have tails of Pareto type and they belong to the class of generalized inverse Gaussian distributions (see, for instance, Section 2.2 of Barndorff-Nielsen and Shephard (2001)). In particular, when $\mu=0, \delta^{2}=n, v=n$, and $n \geq 1$ is an integer, the gamma-distributed random variable $\gamma_{n / 2, n / 2} \stackrel{\mathrm{D}}{=}(1 / n) \gamma_{n / 2,1 / 2} \stackrel{\mathrm{D}}{=}(1 / n) \chi_{n}^{2}$, where $\chi_{n}^{2}$ has a chi-squared distribution with $n$ degrees of freedom, and the $t$-variable $X \sim T\left(n, n^{1 / 2}, 0\right)$ has the representation $X \stackrel{\mathrm{D}}{=} \varepsilon /\left(\chi_{n}^{2} / n\right)^{1 / 2}, \varepsilon \sim N(0,1)$, which is a classical $t$-distribution with $n$ degrees of freedom.

From (2.1), it follows that both the left- and the right-hand tails of the $t$-distribution $T(\nu, \delta, \mu)$ decrease like $|x|^{-v-1}$. The expectation exists when $v>1$, the variance when $v>2$, and the $n$th moments when $v>n$.

From (2.2), we obtain the characteristic function of the $t$-variable $X \sim T(\nu, \delta, \mu)$ :

$$
\phi_{X}(\zeta)=\mathrm{E}\{\exp [\mathrm{i} \zeta X]\}=\mathrm{e}^{\mathrm{i} \zeta \mu} \int_{0}^{\infty} \mathrm{e}^{-\left(\zeta^{2} / 2\right) x} f_{\sigma^{2}}(x) \mathrm{d} x
$$

Here, from (2.4),

$$
f_{\sigma^{2}}(x)=\frac{\left(\frac{1}{2} \delta^{2}\right)^{v / 2}}{\Gamma\left(\frac{1}{2} \nu\right)} x^{-v / 2-1} \mathrm{e}^{-\delta^{2} / 2 x}, \quad x>0,
$$

is the PDF of $\operatorname{R} \Gamma\left(\frac{1}{2} v, \frac{1}{2} \delta^{2}\right)$.

The characteristic function (2.5) can be expressed in terms of the modified Bessel function of the third kind, $K_{\lambda}(x)$ (see Appendix A). From (2.5), (2.6), and (A.1) we arrive at the elegant expression

$$
\phi_{X}(\zeta)=\mathrm{e}^{\mathrm{i} \zeta \mu} \frac{K_{v / 2}(\delta|\zeta|)}{\Gamma\left(\frac{1}{2} v\right)}(\delta|\zeta|)^{v / 2} 2^{1-v / 2}, \quad \zeta \in \mathbb{R} .
$$

For $\zeta=0,(2.7)$ is interpreted in the sense of the asymptotic relation (A.2).

In particular, for $v=1$, the Bessel function $K_{1 / 2}$ has a closed form given by (A.3) and we arrive at the characteristic function of the symmetric Cauchy distribution.

Remark 2.2. The characteristic function of the $t$-distribution has been a topic of some controversy and difficulty (see Dreier and Kotz (2002) for a survey of the available results and discussion). We only note that Dreier and Kotz (2002) developed the alternative expression

$$
\phi(\zeta)=\frac{2^{n} n^{n / 2}}{\Gamma(n)} \int_{0}^{\infty} \mathrm{e}^{-\sqrt{n}(2 x+|\zeta|)}(x(x+|\zeta|))^{(n-1) / 2} \mathrm{~d} x, \quad \zeta \in \mathbb{R},
$$


for the characteristic function of $T\left(n, n^{1 / 2}, 0\right)$, with integer $n \geq 1$, while Jurek (2001) proved (2.7) in the special case of the $T\left(2 v,(2 v)^{1 / 2}, 0\right)$ distribution. Thus, $(2.7)$ is the most general form, to the authors' knowledge (see also Seneta (2004)).

Remark 2.3. Sørensen and Bibby (2003) have used the Laplace transform of the asymmetric scaled $t$-distribution with PDF

$$
\frac{\delta^{\nu}}{\sqrt{\pi} 2^{(\nu-1) / 2} \Gamma\left(\frac{1}{2} \nu\right)} \frac{K_{(\nu+1) / 2}\left(|\beta| \sqrt{\delta^{2}+(x-\mu)^{2}}\right)}{\left(\sqrt{\delta^{2}+(x-\mu)^{2}} /|\beta|\right)^{(\nu+1) / 2}} \mathrm{e}^{\beta(x-\mu)}, \quad x \in \mathbb{R},
$$

where $\nu>0, \delta>0, \beta \in \mathbb{R}$, and $\mu \in \mathbb{R}$. By (A.2), the PDF (2.8) reduces to (2.1) when $\beta=0$.

The Laplace transform of (2.8) is

$$
L T_{X}(\zeta)=\mathrm{e}^{\mu \zeta} \frac{(-\delta \zeta(\zeta+2 \beta))^{v / 2} K_{v / 2}(-\delta \zeta(\zeta+2 \beta))}{\Gamma\left(\frac{1}{2} v\right) 2^{v / 2-1}}
$$

with domain $-2 \beta<\zeta \leq 0$ when $\beta>0$ and $0 \leq \zeta<-2 \beta$ when $\beta<0$. When $\beta=0$, the domain is the set $\{0\}$. Thus, the Laplace transform (and moment generating function) of the symmetric scaled $t$-distribution $T(v, \delta, \mu)$ does not exist. Therefore, we cannot use results from the theory of generalized hyperbolic distributions, as these are based on the Laplace transform. Instead, for the symmetric scaled $t$-distribution, the characteristic function (2.7) can be used. From (A.3), it follows that when $\beta$ is positive, the left-hand tail of (2.8) decreases like $|x|^{-(\nu / 2+1)} \mathrm{e}^{2 \beta x}$ (i.e. is 'semiheavy tailed'), while the right-hand tail decreases like $|x|^{-(\nu / 2+1)}$ (i.e. is 'heavy tailed'). When $\beta$ is negative, the behaviour of the two tails is interchanged. The expectation exists provided that $v>2$, and the $n$th moment exists provided that $v>2 n$.

However, it is possible to obtain the Lévy-Khinchin representation of the characteristic function of the symmetric $t$-distribution $T(\nu, \delta, \mu)$ directly from the results of Halgreen (1979), by choosing $\alpha=|\beta|=0, \delta>0$, and $\lambda=-\frac{1}{2} \nu<0$. We obtain

$$
\log \phi(\zeta)=\mathrm{i} \zeta \mu+\int_{\mathbb{R}}\left(\mathrm{e}^{\mathrm{i} \zeta x}-1-\mathrm{i} \zeta x\right) g(x) \mathrm{d} x,
$$

with

$$
g(x)=\frac{1}{|x|} \int_{0}^{\infty} \frac{\mathrm{e}^{-|x| \sqrt{2 y}} \mathrm{~d} y}{\pi^{2} y\left(J_{v / 2}^{2}(\delta \sqrt{2 y})+Y_{v / 2}^{2}(\delta \sqrt{2 y})\right)},
$$

where $J_{v}(x)$ and $Y_{v}(x)$ respectively denote the Bessel functions of the first and second kinds.

It is known that

$$
x^{2} g(x)=\frac{\delta}{\pi}+\frac{1-v}{4}|x|+o(|x|) \quad \text { as } x \rightarrow 0 .
$$

We see that the Lévy measure of a $t$-distribution $T(v, \delta, \mu)$ has infinite mass in every neighbourhood of the origin.

\subsection{Infinite divisibility and self-decomposability}

It is known that the generalized hyperbolic distributions are infinitely divisible (i.d.) and selfdecomposable (s.d.) (see Barndorff-Nielsen and Halgreen (1977), Halgreen (1979), BarndorffNielsen and Shephard (2001), and the references therein). Thus, the $t$-distributions have to be i.d. and s.d. An independent proof of the infinite divisibility of the $t$-distribution $T\left(n, n^{1 / 2}, 0\right)$, 
with integer $n \geq 1$, was given by Grosswald (1976), while Jurek (2001) proved that the general $t$-distribution $T(\nu, \delta, \mu)$ is both s.d. and i.d.

Recall (see Barndorff-Nielsen and Shephard (2001), for example) that a random variable $X$ is s.d. if its characteristic function $\phi(\zeta)$ has the property that, for every $c \in(0,1)$, there exists a characteristic function $\phi_{c}(\zeta)$ such that

$$
\phi(\zeta)=\phi(c \zeta) \phi_{c}(\zeta)
$$

for all $\zeta \in \mathbb{R}$. In terms of random variables, property (2.9) means that, for any $c \in(0,1)$, there exists a random variable $X_{c}$ such that

$$
X \stackrel{\mathrm{D}}{=} c X+X_{c}
$$

and $X$ and $X_{c}$ are independent.

It is known that all s.d. characteristic functions (or random variables or probability distributions) are i.d.; that is, for every $n \geq 1$, there exists a characteristic function $\phi_{n}(\zeta), \zeta \in \mathbb{R}$, such that $\phi(\zeta)=\left[\phi_{n}(\zeta)\right]^{n}, \zeta \in \mathbb{R}$.

A stochastically continuous process $L(t), t \geq 0$, with $L(0)=0$ and strictly stationary and independent increments, is called a (homogeneous) Lévy process. We may, without loss of generality, choose a version with càdlàg paths (continuous from the right with left limits) or with paths almost surely from the Skorokhod space of càdlàg functions (see, for example, Bertoin (1996, p. 18) or Sato (1999)), with the strong Markov property (Bertoin (1996, p. 20)). The law of $L(t)$ is then determined by the law of $L(1)$, which is i.d. The independence and stationarity of the increments of the Lévy process mean that the cumulant transform is given by

$$
\kappa_{L(t)}(\zeta)=t \kappa_{L(1)}(\zeta), \quad \zeta \in \mathbb{R} .
$$

Familiar special classes of Lévy process are Brownian motion and the compound Poisson processes. For every i.d. random variable $T$, there exists a Lévy process such that $L(1) \stackrel{\mathrm{D}}{=} X$. All Lévy processes except for Brownian motion have jumps.

We say that $X(t)$ has the scaling property if, for each $c, 0<c<1$, there exists a nonrandom function $M(c)$ such that

$$
X(c t) \stackrel{\mathrm{D}}{=} M(c) X(t), \quad t \geq 0 .
$$

For instance, Brownian motion (a Lévy process) has the scaling property (2.10) with $M(c)=c^{1 / 2}$, while fractional Brownian motion, which is not a Lévy process, has the scaling property (2.10) with $M(c)=c^{H}, 0<H<1$. The symmetric stable Lévy process $L(t), t \geq 0$, with $\kappa_{L(1)}(\zeta)=|\zeta|^{\alpha}, 0<\alpha \leq 2$, has the scaling property (2.10) with $M(c)=c^{1 / \alpha}$ (see Samorodnitsky and Taqqu (1994) or Mandelbrot (2001a), (2001b) for details).

The following theorem was proved by Jurek (2001).

Theorem 2.1. Let $L(t)$ be a Lévy process with the strong Markov property. That is, for any independent random variable $T \geq 0, L(t+T)-L(T)$ and $L(t)$ have the same probability distributions. We assume that $L(t)$ has the scaling property (2.10). If $T$ is s.d. and the (nonrandom) scaling function $M(c)$ is a homeomorphism of the unit interval, then $L(T)$ is s.d.

From (2.2) and Theorem 2.1, we obtain a second theorem (see Jurek (2001) for details).

Theorem 2.2. The $t$-distribution $T(\nu, \delta, \mu)$ with PDF (2.1) and characteristic function (2.9) is s.d. (and i.d.). 
From Theorem 2.2, it follows that there exists a Lévy process $L_{S}(t), t \geq 0$, such that the random variable $L_{S}(1)$ has the symmetric scaling $t$-distribution $T(\nu, \delta, \mu)$ with density function (2.1) and characteristic function (2.7).

For $v>1, \mathrm{E}\left\{L_{S}(t)\right\}=t \mu, t \geq 0$, and $L_{S}(t)=t \mu+L_{S}^{0}(t), t \geq 0$, where $\mathrm{E}\left\{L_{S}^{0}(t)\right\}=0$.

The PDF of the random variable $L_{S}^{0}(t), t \geq 0$, takes the form

$$
\tilde{f}_{t}(x)=\frac{2^{t(1-v / 2)}}{\sqrt{\pi} \Gamma^{t}\left(\frac{1}{2} \nu\right)} \int_{0}^{\infty} \cos (\zeta x)(\delta|\zeta|)^{\nu t / 2} K_{\nu / 2}^{t}(\delta|\zeta|) \mathrm{d} \zeta
$$

and it is not easy to compute except in the case $t=1$, in which (2.11) reduces to (2.1) with $\mu=0$. However, the asymptotic behaviour of $f(x)$, the PDF of $L_{s}$, for $x \rightarrow 0$ or $x \rightarrow \infty$ is obtainable by using Tauberian-Abelian-type results for the Fourier transforms (see, for example, Bingham et al. (1987)) and (A.2) and (A.3).

\subsection{The nonlinear Fokker-Planck equation and entropy}

The importance of the $t$-distribution in statistical physics has been highlighted in Tsallis et al. (1995), Tsallis and Bukman (1996), and Vignat and Bercher (2003) (also see the references therein). An application of these results to finance has been provided by Borland (2002).

The point of departure from Gaussian maximum entropy approaches (in the sense of the Boltzmann-Gibbs-Shannon entropy) is the feature that the entropy used is the nonextensive (Havdra-Charvát (1967) or Tsallis) entropy for a time-dependent PDF $f_{t}(x)$ :

$$
H_{q}=-\frac{1}{1-q}\left(1-\int_{\mathbb{R}} f_{t}^{q}(x) \mathrm{d} x\right), \quad x \in \mathbb{R}, q \in \mathbb{R} \backslash\{0\} .
$$

The parameter $q$ characterizes the nonextensivity of the entropy. In the limit $q \rightarrow 1$, the Havdra-Charvát or Tsallis entropy becomes the Boltzmann-Gibbs-Shannon entropy

$$
H_{1}=-\int_{\mathbb{R}} f_{t}(x) \log f_{t}(x) \mathrm{d} x .
$$

Maximizing the entropy (2.12), subject to the constraints

$$
\int_{\mathbb{R}} f_{t}(x) \mathrm{d} x=1, \quad \int_{\mathbb{R}}(x-\bar{x}(t)) f_{t}^{q}(x) \mathrm{d} x=0, \quad \int_{\mathbb{R}}(x-\bar{x}(t))^{2} f_{t}^{q}(x) \mathrm{d} x=\sigma_{q}^{2}(t),
$$

for fixed $q$ and specified functions $\bar{x}(t)$ and $\sigma_{q}^{2}(t)$, yields

$$
f_{t}(x)=\frac{1}{Z(t)} \frac{1}{\left[1+\beta(t)(q-1)(x-\bar{x}(t))^{2}\right]^{1 /(q-1)}}, \quad x \in \mathbb{R},
$$

where

$$
Z(t)=\frac{B\left(\frac{1}{2}, 1 /(q-1)-\frac{1}{2}\right)}{\sqrt{(q-1) \beta(t)}},
$$

$B(\cdot, \cdot)$ being the beta function.

The variance of the distribution (2.14) is

$$
\sigma_{q}^{2}(t)= \begin{cases}\frac{1}{(5-3 q) \beta(t)}, & q<\frac{5}{3} \\ \infty, & q \geq \frac{5}{3}\end{cases}
$$


Hence, for applications of data of finite variance, the parameter $q$ satisfies

$$
1 \leq q<\frac{5}{3}
$$

The distribution (2.14) is a time-dependent generalization of the $t$-distribution (2.1) with an appropriate reparametrization.

An important property of the PDF (2.14) is that it is the solution of a nonlinear Fokker-Planck (or Kolmogorov) equation, i.e.

$$
\frac{\partial}{\partial t} f_{t}(x)=-\frac{\partial}{\partial x}\left[f_{t}(x) F(x)\right]+\frac{D}{2} \frac{\partial^{2}}{\partial x^{2}} f_{t}^{2-q}(x),
$$

where $D$ is a constant.

The PDF (2.14) solves partial differential equation (2.15) with driving term $F(x)=a-b x$ if the time-dependent parameters are given by

$$
\begin{gathered}
-\frac{1}{3-q} \frac{\mathrm{d} Z^{3-q}(t)}{\mathrm{d} t}+2(2-q) D \beta\left(t_{0}\right) Z^{2}\left(t_{0}\right)-b Z^{3-q}(t)=0, \\
\frac{\beta(t)}{\beta\left(t_{0}\right)}=\left[\frac{Z(t)}{Z\left(t_{0}\right)}\right]^{2}, \quad \frac{\mathrm{d} \bar{x}}{\mathrm{~d} t}=a-b \bar{x},
\end{gathered}
$$

or

$$
\begin{aligned}
\beta(t)^{(q-3) / 2}= & \beta\left(t_{0}\right)^{(q-3) / 2} \exp \left[b(q-3)\left(t-t_{0}\right)\right] \\
& -2 D b^{-1}(2-q)\left[\beta\left(t_{0}\right) Z^{2}\left(t_{0}\right)\right]^{(q-1) / 2}\left(\exp \left[-b(3-q)\left(t-t_{0}\right)\right]-1\right) .
\end{aligned}
$$

In the limit $q \rightarrow 1$, the standard linear diffusion (or heat) equation is recovered and the $t$-distribution (2.14) becomes Gaussian, while the Tsallis entropy becomes the BoltzmannGibbs-Shannon entropy (2.13) and the constraints correspond to the Gaussian maximum entropy principle.

The Rényi (1961) entropy (of the multidimensional PDF $f(\boldsymbol{x}), \boldsymbol{x} \in \mathbb{R}^{n}$ )

$$
\tilde{H}_{\alpha}=\frac{1}{1-\alpha} \log \int_{\mathbb{R}^{n}} f^{\alpha}(\boldsymbol{x}) \mathrm{d} \boldsymbol{x}, \quad \boldsymbol{x} \in \mathbb{R}^{n}, \alpha \in \mathbb{R} \backslash\{0\},
$$

is also a generalization of the Boltzmann-Gibbs-Shannon entropy

$$
H_{1}=-\int_{\mathbb{R}^{n}} f(\boldsymbol{x}) \log f(\boldsymbol{x}) \mathrm{d} \boldsymbol{x}
$$

and converges to it as $\alpha \rightarrow 1$.

The random vector $\boldsymbol{X}=\left(X_{1}, \ldots, X_{n}\right)^{\top}$ is said to have a multidimensional Student distribution $T(\nu, \boldsymbol{\Sigma}, \boldsymbol{\mu})$, with mean $\boldsymbol{\mu} \in \mathbb{R}^{n}$, scaling matrix $\boldsymbol{\Sigma}>0$, and $v$ degrees of freedom, if its density is given by

$$
\begin{aligned}
f_{v}(\boldsymbol{x})= & (v \pi)^{-n / 2} \Gamma\left(\frac{1}{2}(n+v)\right) \Gamma^{-1}\left(\frac{1}{2} v\right)|\boldsymbol{\Sigma}|^{-1 / 2} \\
& \times\left[1+(\boldsymbol{x}-\boldsymbol{\mu})^{\top}[v \boldsymbol{\Sigma}]^{-1}(\boldsymbol{x}-\boldsymbol{\mu})\right]^{-(n+v) / 2}, \quad \boldsymbol{x} \in \mathbb{R}^{n} .
\end{aligned}
$$

The characteristic function of the Student distribution $T(\nu, \Sigma, \mu)$ is of the form

$$
\phi(\zeta)=\mathrm{E}\left\{\mathrm{e}^{\mathrm{i}\langle\zeta, X\rangle}\right\}=\mathrm{e}^{\mathrm{i}\langle\zeta, \mu\rangle} \frac{K_{\nu / 2}\left(\sqrt{\nu \zeta^{\top} \Sigma \zeta}\right)}{\Gamma\left(\frac{1}{2} v\right)}\left(\sqrt{\nu \zeta^{\top} \Sigma \zeta}\right)^{v / 2} 2^{1-v / 2}, \quad \zeta \in \mathbb{R}^{n},
$$


and the Rényi entropy

$\tilde{H}_{\alpha}=\frac{1}{1-\alpha} \log \frac{B\left(\frac{1}{2}[\alpha(n+v)-n], \frac{1}{2} n\right)}{B^{\alpha}\left(\frac{1}{2} v, \frac{1}{2} n\right)}+\frac{1}{2} \log \left[(v \pi)^{n}|\Sigma|\right]-\log \Gamma\left(\frac{1}{2} n\right), \quad \alpha>\frac{n}{n+v}$,

converges, as $v \rightarrow \infty$, to the Rényi entropy

$$
\log \left[(2 \pi)^{n / 2}|\Sigma|^{1 / 2}\right]-\frac{n}{2(1-\alpha)} \log \alpha
$$

of the multidimensional normal distribution $N(\boldsymbol{\mu}, \boldsymbol{\Sigma})$.

The Student distribution $T(v,[(v-2) / v] \boldsymbol{\Sigma}, \mathbf{0})$ with $v=2 /(1-\alpha)-n>2$ is also the maximum Rényi entropy distribution under the constraints

$$
\mathrm{E}\left\{\boldsymbol{X} \boldsymbol{X}^{\top}\right\}=\boldsymbol{\Sigma} \text { and } \quad \frac{n}{n+2}<\alpha<1
$$

(see Costa et al. (2003)). It can be seen, from the nonsymmetric Bregman divergence measure, that

$$
0 \leq D(f, g)=\operatorname{sgn}(\alpha-1) \int_{\mathbb{R}^{n}}\left[\frac{1}{\alpha} f^{\alpha}(\boldsymbol{x})+\frac{\alpha-1}{\alpha} g^{\alpha}(\boldsymbol{x})-f(\boldsymbol{x}) g^{\alpha-1}(\boldsymbol{x})\right] \mathrm{d} \boldsymbol{x}
$$

(which is 0 if and only if $g=f$ ).

\section{Stationary processes of Student type}

In this section, we shall introduce several classes of stationary stochastic process with marginal symmetric scaled Student distribution $T(\nu, \delta, \mu)$.

\subsection{Student Ornstein-Uhlenbeck-type processes}

Based on the theory of non-Gaussian Ornstein-Uhlenbeck-based models (see BarndorffNielsen et al. (1998), Barndorff-Nielsen and Shephard (2001), and references therein) we can introduce the Student Ornstein-Uhlenbeck-type (OU-type) processes.

The key result is known as the random integral representation (see Jurek and Mason (1993, Theorem 3.9.3) and the bibliographic comments there, and Barndorff-Nielsen and Shephard (2001, Equation (12)); see also Sato (1999, Theorem 17.5)).

Theorem 3.1. The random variable $X$ has an s.d. distribution if and only if there exists a Lévy process $Y(t)$ such that

$$
\mathrm{E}\{\log (1+|Y(1)|)\}<\infty \quad \text { and } X \stackrel{\mathrm{D}}{=} \int_{0}^{\infty} \mathrm{e}^{-s} \mathrm{~d} Y(s) .
$$

Then $Y$ is unique in distribution. Moreover, if the cumulant transform $\kappa_{X}(\zeta)$ is differentiable for $\zeta \neq 0$ and $\zeta \kappa_{X}(\zeta) \rightarrow 0$ for $0 \neq \zeta \rightarrow 0$, then we have

$$
\kappa_{Y(1)}(\zeta)=\zeta \frac{\mathrm{d}}{\mathrm{d} \zeta} \kappa_{X}(\zeta)
$$

The process $Y(t)$ is referred to as the background driving Lévy process (BDLP) for $X$. 
From (2.7), (3.1), and (A.1) we obtain the following expression for the cumulant transform of the BDLP $Y(t)$ for the $t$-distribution $T(\nu, \delta, \mu)$ with characteristic function (2.9), with $\kappa_{Y(1)}(0)=0$ :

$$
\kappa_{Y(1)}(\zeta)=\log \mathrm{E}\{\exp [\mathrm{i} \zeta Y(1)]\}=\mathrm{i} \zeta \mu-\delta|\zeta| \frac{K_{\nu / 2-1}(\delta|\zeta|)}{K_{\nu / 2}(\delta|\zeta|)}, \quad \zeta \in \mathbb{R}, \quad \zeta \neq 0
$$

Note that, for the special case of the $t$-distribution $T\left(2 v,(2 v)^{1 / 2}, 0\right),(3.2)$ was proved by Jurek (2001) using a different method.

A stochastic process $X(t)$ is said to be of OU type if it satisfies a stochastic differential equation (SDE) of the form

$$
\mathrm{d} X_{t}=-\lambda X_{t} \mathrm{~d} t+\mathrm{d} Y(t)
$$

where $Y(t)$ is the BDLP.

From our Theorem 3.1 and Theorem 1 of Barndorff-Nielsen and Shephard (2001), by using (3.2) we arrive at the following statement.

Theorem 3.2. There exists a stationary (in the strict sense) stochastic process $X_{t}, t \in \mathbb{R}$, which has marginal $t$-distribution $T(\nu, \delta, \mu)$ with density function $(2.1)$ and $B D L P Y(t)$ with cumulant transform (3.2), such that $X_{t}$ satisfies the $\operatorname{SDE}(3.3)$ for all $\lambda>0$;

$$
\begin{aligned}
X_{t} & =\mathrm{e}^{-\lambda t} X_{0}+\mathrm{e}^{-\lambda t} \int_{0}^{t} \mathrm{e}^{\lambda s} \mathrm{~d} Y(\lambda s) \\
& =\int_{-\infty}^{t} \mathrm{e}^{-\lambda(t-s)} \mathrm{d} Y(\lambda s), \quad \lambda>0, t \in \mathbb{R} ; \quad \text { and }
\end{aligned}
$$

(ii) if $v>1$ then $\mathrm{E}\left\{X_{t}\right\}=\mu$, and if $v>2$ then the correlation function is given by

$$
\nu(\tau)=\operatorname{corr}\left(X_{t+\tau}, X_{t}\right)=\mathrm{e}^{-\lambda|\tau|}, \quad \tau \in \mathbb{R} .
$$

The stationary process $X_{t}, t \in \mathbb{R}$, can be referred to as the Student OU-type process.

Remark 3.1. The higher-order cumulant functions of the stochastic process (3.4) and the higher-order spectral densities of this process can be obtained (see Anh et al. (2002) for details).

In the same manner, the stationary autoregressive processes with marginal $t$-distributions can be constructed on the basis of the self-decomposability of the $t$-distributions (see Theorem 2.2). Let $X_{0}=X$ be a random variable with a $t$-distribution $T(\nu, \delta, \mu)$ and define a stationary firstorder autoregressive sequence $X_{n}, n \geq 1$, such that

$$
X_{n}=c X_{n-1}+\varepsilon_{n}, \quad n \geq 1
$$

where $0<c<1$ and $\left\{\varepsilon_{n}\right\}_{n=0}^{\infty}$ are independent, identically distributed random variables (comprising a so-called innovation process) independent of $\left\{X_{n}\right\}_{n=1}^{\infty}$. In distribution, we have

$$
X_{0}=c X_{0}+\varepsilon_{1}
$$

Hence, if (3.5) holds for all $c \in(0,1)$, then $X_{0}$ is an s.d. random variable (see (2.9)). 
In other words, the s.d. Student distribution $X_{0} \sim S(v, \delta, \mu)$ can be realized as the marginal distribution of an autoregressive sequence. In fact, written in terms of the $\operatorname{BDLP} Y(t)$ of $X_{0}$,

$$
\varepsilon_{1} \stackrel{\mathrm{D}}{=} \int_{0}^{-\log c} \mathrm{e}^{-s} \mathrm{~d} Y(s)
$$

i.e. the cumulant transform of the BDLP $Y(t)$, which is given by (3.2).

Thus, there exists a stationary (in the strict sense) solution $X_{n}$ of the autoregressive equation (3.5), with the following properties:

$$
\begin{array}{cl}
X_{n} \sim T(\nu, \delta, \mu), \quad \mathrm{E}\left\{X_{n}\right\}=\mu & (\text { if } v>1), \\
\operatorname{corr}\left(X_{n}, X_{n+\tau}\right)=c^{|\tau|}, \quad 0<c<1, \tau=0, \pm 1, \ldots & (\text { if } v>2) .
\end{array}
$$

Remark 3.2. It follows from Barndorff-Nielsen (1998) or Barndorff-Nielsen and Shephard (2001) that there exists a BDLP $\tilde{Y}(t), t \geq 0$, and a stationary OU process $\tilde{X}_{t} \sim \mathrm{R} \Gamma\left(\frac{1}{2} \nu, \frac{1}{2} \delta^{2}\right)$ such that $\tilde{X}_{t}$ is a solution to an SDE similar to (3.3), with $\mathrm{E}\left\{\tilde{X}_{t}\right\}=\frac{1}{2} \delta^{2} /\left(\frac{1}{2} v-1\right)$ when $v>2$ and

$$
\operatorname{corr}\left(\tilde{X}_{t}, \tilde{X}_{t+\tau}\right)=\mathrm{e}^{-\lambda|\tau|}, \quad \tau \in \mathbb{R}, \quad \text { when } v>4 .
$$

The characteristic function of the $\mathrm{R} \Gamma\left(\frac{1}{2} \nu, \frac{1}{2} \delta^{2}\right)$ distribution is of the form (see Witkovsky (2002))

$$
\mathrm{E}\left\{\mathrm{e}^{\mathrm{i} \zeta \tilde{X}_{t}}\right\}=\frac{2\left(\frac{1}{2} \delta\right)^{v / 2}}{\Gamma\left(\frac{1}{2} \nu\right)}\left(-\mathrm{i} \zeta \frac{2}{\delta^{2}}\right)^{v / 4} K_{\nu / 2}\left(\delta^{2}\left(-\mathrm{i} \zeta \frac{2}{\delta^{2}}\right)^{1 / 2}\right), \quad \zeta \in \mathbb{R},
$$

and, by (3.1), the BDLP $\tilde{Y}(t), t \geq 0$, in an SDE of type (3.3) for the $\mathrm{R} \Gamma\left(\frac{1}{2} v, \frac{1}{2} \delta^{2}\right)$ distribution has the cumulant function

$$
\kappa_{\tilde{Y}(1)}(\zeta)=\frac{\mathrm{i} \zeta}{\left(-\mathrm{i} \zeta 2 / \delta^{2}\right)^{1 / 2}} \frac{K_{\nu / 2-1}\left(\delta^{2}\left(-\mathrm{i} \zeta 2 / \delta^{2}\right)^{1 / 2}\right)}{K_{\nu / 2}\left(\delta^{2}\left(-\mathrm{i} \zeta 2 / \delta^{2}\right)^{1 / 2}\right)}, \quad \zeta \neq 0,
$$

with $\kappa_{\tilde{Y}(1)}(0)=0$.

Let us consider a Gaussian OU process $Z_{t}$, independent of $\tilde{X}_{t}$, with mean 0 and correlation function $\operatorname{corr}\left(Z_{t}, Z_{t+\tau}\right)=\mathrm{e}^{-\lambda|\tau|}, \tau \in \mathbb{R}$. The stationary process $\bar{X}_{t}=Z_{t} \tilde{X}_{t}^{1 / 2}, t \in \mathbb{R}$, then has a marginal Student distribution, but this process is different from the Student-type process that was constructed in Theorem 3.2.

Remark 3.3. It follows from Ait-Sahalia (1996) and Bibby et al. (2003) that a stationary process with PDF (2.1) and correlation function $\operatorname{corr}\left(X_{t}, X_{t+\tau}\right)=\mathrm{e}^{-\lambda|\tau|}, \tau \in \mathbb{R}$, can be obtained as a unique Markovian weak solution, with invariant PDF (2.1), to the SDE

$$
\mathrm{d} X_{t}=-\lambda\left(X_{t}-\mu\right) \mathrm{d} t+\sqrt{\frac{2 \lambda \delta^{2}}{v-1}\left[1+\left(\frac{X_{t}-\mu}{\delta}\right)^{2}\right]} \mathrm{d} W(t), \quad v>2,
$$

where $W(t), t \in \mathbb{R}$, is a standard Brownian motion. This process has continuous paths, in contrast to the process described in Theorem 3.2.

On the other hand, the unique Markovian solution with invariant $\operatorname{PDF} \operatorname{R} \Gamma\left(\frac{1}{2} \nu, \frac{1}{2} \delta^{2}\right)$ can be obtained in a similar way from the SDE

$$
\mathrm{d} \tilde{X}_{t}=-\lambda\left(\tilde{X}_{t}-\frac{\delta^{2}}{v-2}\right) \mathrm{d} t+\sqrt{\frac{4 \lambda}{v-2} \tilde{X}_{t}^{2}} \mathrm{~d} W(t), \quad t \geq 0, \delta>0, v>2,
$$

whence $X_{t}=v+\tilde{X}_{t} Z_{t} \sim T\left(\mu, \delta, \delta^{2} /(v-2)\right)$, where $Z_{t}$ is a Gaussian OU process independent of $\tilde{X}_{t}$. 
Remark 3.4. Tarami and Pourahmadi (2003) have shown that if $\boldsymbol{\varepsilon}=\left(\varepsilon_{1}, \ldots, \varepsilon_{n}\right)$ follows a Student $T\left(v, \sigma^{2} \boldsymbol{I}_{n}, \mathbf{0}\right)$ distribution, where $\boldsymbol{I}_{n}$ is the $n \times n$ identity matrix, then the stationary solution to the autoregressive equation

$$
X_{t}=\sum_{k=1}^{p} c_{k} X_{t-k}+\varepsilon_{t}, \quad t=0, \pm 1, \ldots,
$$

is such that, for every $n \geq 1$, the vector $\boldsymbol{X}=\left(X_{1}, \ldots, X_{n}\right)$ has Student $T(v, \boldsymbol{\Sigma}, \mathbf{0})$ distribution with PDF (2.16) with $\boldsymbol{\mu}=\mathbf{0}$, and the autocovariance matrix $\boldsymbol{\Gamma}_{n}$ of $\boldsymbol{X}$ is given by $\boldsymbol{\Gamma}_{n}=$ $([v-2] / v)^{-1} \Sigma, v>2$. Note that the entries of $\boldsymbol{\varepsilon}=\left(\varepsilon_{1}, \ldots, \varepsilon_{n}\right)$ are uncorrelated but not independent and that, when $\varepsilon_{1}, \ldots, \varepsilon_{n}$ are independent, each with the univariate Student distribution (2.1), the joint distribution of $\varepsilon$ is not a multivariate Student distribution with PDF (2.16).

In this subsection, stationary processes of Student type with discrete- or continuous-time and short-range dependence have been constructed. In the next subsection, we shall introduce stationary processes of Student type with long-range dependence.

\subsection{Stationary Student processes with long-range dependence}

In this subsection, we propose some models for strictly stationary processes with long-range dependence and marginal $t$-distributions. These are based on the results of Barndorff-Nielsen and Pérez-Abreu (1999), which use the theory of multidimensional type-G distributions (see, for instance, Rosiński (1991)).

Any real second-order stationary process $X_{t}, t \in \mathbb{R}$, which is continuous in the squared mean and has mean $\mu \in \mathbb{R}$, is representable as

$$
X_{t}=\mu+\int_{\mathbb{R}} \cos (\lambda t) v(\mathrm{~d} \lambda)+\int_{\mathbb{R}} \sin (\lambda t) w(\mathrm{~d} \lambda),
$$

where $v(t), t \in \mathbb{R}$, and $w(t), t \in \mathbb{R}$, are mutually orthogonal zero-mean, square-integrable real processes with orthogonal increments, and the stochastic integrals in (3.7) are defined in the $L^{2}$ sense (see, for instance, Cramér and Leadbetter (1967, p. 137)). The correlation function of (3.7) is given by

$$
R(\tau)=\int_{\mathbb{R}} \cos (\lambda \tau) F(\mathrm{~d} \lambda)
$$

with

$$
F(\mathrm{~d} \lambda)=\frac{1}{2} \mathrm{E}\left\{\cos ^{2}(\lambda t) v(\mathrm{~d} \lambda)^{2}+\sin ^{2}(\lambda t) w(\mathrm{~d} \lambda)^{2}\right\} .
$$

Let us consider a special case that corresponds to a bivariate innovation process $(v(t), w(t))$ of the form

$$
(v(t), w(t))=z(F(\lambda))=\left[z_{1}(F(\lambda)), z_{2}(F(\lambda))\right],
$$

where $z(\lambda)=\left[z_{1}(\lambda), z_{2}(\lambda)\right]$ is a bivariate Lévy process such that $z(1)$ is of type G. That is, $z(1) \stackrel{\text { D }}{=} \sigma \boldsymbol{\varepsilon}$, where $\sigma>0$ and $\boldsymbol{\varepsilon}$ are independent with $\boldsymbol{\varepsilon} \sim N_{2}\left(0, \boldsymbol{I}_{2}\right)$ (the standard twodimensional normal distribution), and $\sigma^{2}$ follows an inverse gamma distribution $\operatorname{R} \Gamma\left(\frac{1}{2} \nu, \frac{1}{2} \delta^{2}\right)$ with $v>4$.

From Barndorff-Nielsen and Pérez-Abreu (1999), it follows that the process (3.8) is well defined, the integrals being interpreted with respect to independently scattered random measures (see Rajput and Rosiński (1989), Kwapien and Woyczyński (1992), or Barndorff-Nielsen 
(2001)). This process is (strictly) stationary and its marginal distribution has a characteristic function of the form (2.5) or (2.7), that is $X_{t} \sim T(\nu, \delta, \mu)$. Moreover, $\mathrm{E}\left\{X_{t}\right\}=\mu$ and the correlation function is given by (3.8), where $F$ is an arbitrary distribution function on $\mathbb{R}$.

Let us consider the special case of the distribution function

$$
F(\mathrm{~d} \lambda)=f_{\alpha, \varkappa}(\lambda) \mathrm{d} \lambda, \quad \lambda \in \mathbb{R}, 0<\alpha \leq 1, \varkappa \in \mathbb{R},
$$

where the spectral density is

$$
f_{\alpha, \varkappa}(\lambda)=\frac{1}{2}\left[f_{\alpha, 0}(\lambda+\varkappa)+f_{\alpha, 0}(\lambda-\varkappa)\right], \quad \lambda \in \mathbb{R},
$$

with

$$
f_{\alpha, 0}(\lambda)=\frac{2^{(1-\alpha) / 2}}{\sqrt{\pi} \Gamma\left(\frac{1}{2} \alpha\right)} K_{(1-\alpha) / 2}(|\lambda|)|\lambda|^{(1-\alpha) / 2} .
$$

From (3.8)-(3.10), we find (see Anh et al. (2004) for details) that the correlation function of the stationary Student process (3.7) takes the form

$$
R(t)=\frac{\cos (\varkappa t)}{\left(1+t^{2}\right)^{\alpha / 2}}, \quad 0<\alpha \leq 1, \varkappa \in \mathbb{R}, t \in \mathbb{R} .
$$

Thus, for $\varkappa=0$ the spectral density (3.10) has the singular property

$$
f_{\alpha, 0}(0)=\infty, \quad 0<\alpha \leq 1,
$$

while, for $\varkappa \neq 0$, we find that

$$
f_{\alpha, \varkappa}( \pm \varkappa)=\infty, \quad 0<\alpha \leq 1
$$

For any $0<\alpha \leq 1$ and $\varkappa \in \mathbb{R}$,

$$
\int_{-\infty}^{\infty}|R(t)| \mathrm{d} t=\infty
$$

that is, the stationary Student process (3.7) with distribution function (3.9) exhibits long-range dependence (see Heyde and Yang (1997) and references therein for a more detailed discussion).

We summarize our results in the following theorem.

Theorem 3.3. For any $v>4$, there exists a strictly stationary process of the form (3.7) with marginal Student distribution $T(\nu, \delta, \mu)$ and the correlation function (3.11) or the spectral density (3.10).

Remark 3.5. Similar results can be obtained for correlation functions of the form

$$
R(t)=\frac{\cos (\varkappa t)}{\left(1+|t|^{\beta}\right)^{\alpha}}, \quad \varkappa \in \mathbb{R}, 0<\alpha \beta \leq 1, t \in \mathbb{R} .
$$

The corresponding spectral densities can be found in Barndorff-Nielsen and Leonenko (2005). 


\subsection{Stationary Student and inverse gamma processes with given dependence structure}

In this subsection, we shall use some special constructions for stationary Student and inverse gamma processes with given (monotonic) correlation function. We make use of some ideas from Berman (1992) and Leonenko (1999, Chapter 2) (see also the references therein).

Let $\eta_{0}(t), \eta_{1}(t), \ldots, \eta_{v}(t), v \geq 1, t \in \mathbb{R}$, be independent copies of a stationary Gaussian process $\eta(t), t \in \mathbb{R}$, with zero mean, unit variance, and continuous monotonic correlation function $\rho_{\eta}(\tau) \geq 0, \tau \in \mathbb{R}$.

We introduce the stationary process

$$
X_{t}=\mu+\frac{\eta_{0}(t)}{\sqrt{2 \chi_{v}^{2}(t) / v}}, \quad t \in \mathbb{R},
$$

where $\mu \in \mathbb{R}$ and the chi-squared process $\chi_{v}^{2}(t)$ is defined by

$$
\chi_{v}^{2}(t)=\frac{1}{2}\left(\eta_{1}^{2}(t)+\cdots+\eta_{v}^{2}(t)\right), \quad t \in \mathbb{R} .
$$

From (3.12) and (3.13), we find that the strictly stationary process (3.12) has marginal $t$-distribution $T\left(\nu, v^{1 / 2}, \mu\right)$.

From Berman (1992) and Leonenko (1999, pp. 109-111), we collect the following properties of the chi-squared process (3.13).

Theorem 3.4. The strictly stationary process (3.13) has the following properties.

$$
\begin{gathered}
\mathrm{E}\left\{\chi_{\nu}^{2}(t)\right\}=\frac{1}{2} v, \quad \operatorname{var}\left\{\chi_{v}^{2}(t)\right\}=\frac{1}{2} \nu, \\
\operatorname{cov}\left(\chi_{\nu}^{2}(t), \chi_{v}^{2}(t+\tau)\right)=\frac{1}{2} v \rho_{\eta}(\tau), \quad \tau \in \mathbb{R} .
\end{gathered}
$$

(ii) The PDF of $\chi_{v}^{2}(t)$ is of the form

$$
p_{\nu / 2}(x)=x^{\nu / 2-1} \frac{\mathrm{e}^{-x}}{\Gamma\left(\frac{1}{2} v\right)}, \quad x>0,
$$

while the bivariate PDF of the random vector $\left(\chi_{v}^{2}(t), \chi_{v}^{2}(t+\tau)\right)$ takes the form

$$
\begin{aligned}
p_{\nu / 2}(x, y ; \gamma)= & \left(\frac{x y}{\gamma}\right)^{(\nu-2) / 4} \mathrm{e}^{-(x+y) /(1-\gamma)} I_{(\nu-2) / 2} \\
& \times\left(2 \frac{\sqrt{x y \gamma}}{1-\gamma}\right) \frac{1}{\Gamma\left(\frac{1}{2} \nu\right)(1-\gamma)}, \quad x, y>0,
\end{aligned}
$$

with $\gamma=\rho_{\eta}^{2}(\tau)$, where $I_{\lambda}(z)$ is the modified Bessel function of the first kind.

(iii) $\mathrm{E}\left\{e_{k}\left(\chi_{v}^{2}(t)\right)\right\}=0, k=1,2, \ldots$, and

$$
\mathrm{E}\left\{e_{k}\left(\chi_{v}^{2}(t)\right) e_{m}\left(\chi_{v}^{2}(t+\tau)\right)\right\}=\delta_{k}^{m} \rho_{\eta}^{2 k}(\tau),
$$

where $\delta_{k}^{m}$ is the Kronecker symbol,

$$
e_{k}(u)=L_{k}^{\nu / 2-1}(u)\left\{k ! \frac{\Gamma\left(\frac{1}{2} v\right)}{\Gamma\left(\frac{1}{2} v+k\right)}\right\}^{1 / 2},
$$

and

$$
L_{k}^{\beta}(u)=\frac{1}{k !} u^{-\beta} \mathrm{e}^{u} \frac{\mathrm{d}^{k}}{\mathrm{~d} u^{k}}\left\{u^{\beta+k} \mathrm{e}^{-u}\right\}
$$

are the generalized Laguerre polynomials of index $\beta$, for $k \geq 0$. 
Note that (3.15) follows from the Hille-Hardy formula (see Bateman and Erdélyi (1953, Chapter 10)), which can be written in the form

$$
p_{\beta}(x, y ; \gamma)=p_{\beta}(x) p_{\beta}(y)\left[1+\sum_{k=1}^{\infty} \gamma^{k} e_{k}(x) e_{k}(y)\right], \quad x, y>0,
$$

with $\beta=\frac{1}{2} \nu$ and $\gamma=\rho^{2}(\tau), 0<\gamma<1$. This means that $\left\{e_{k}(x)\right\}_{k=0}^{\infty}$ is a complete orthogonal system of functions in the Hilbert space $L_{2}\left((0, \infty), p_{v / 2}(x) \mathrm{d} x\right)$ (see, e.g. Courant and Hilbert (1953, Section II.9.4) or Leonenko (1999, pp. 103-112)). Thus, for a nonrandom function

$$
G(x) \in L_{2}\left((0, \infty), p_{v / 2}(x) \mathrm{d} x\right),
$$

the following expansion holds:

$$
\begin{gathered}
G(x)=\sum_{k=1}^{\infty} C_{k} e_{k}(x), \quad C_{k}=\int_{0}^{\infty} G(x) p_{v / 2}(x) e_{k}(x) \mathrm{d} x, \\
\sum_{k=1}^{\infty} C_{k}^{2}=\int_{0}^{\infty} G^{2}(x) p_{v / 2}(x) \mathrm{d} x<\infty
\end{gathered}
$$

Let us consider the strictly stationary process

$$
r(t)=\left[\frac{2}{v} \chi_{v}^{2}(t)\right]^{-1}, \quad t \in \mathbb{R}
$$

whose marginal distribution is inverse gamma $\mathrm{R} \Gamma\left(\frac{1}{2} v, \frac{1}{2} v\right)$ since $\chi_{v}^{2}(t) \sim \Gamma\left(\frac{1}{2} v, 1\right)$ (see Remark 2.1).

Note that $r(t)=G\left(\chi_{v}^{2}(t)\right)$, with

$$
G(x)=\frac{v}{2 x} \in L_{2}\left((0, \infty), p_{v / 2}(x) \mathrm{d} x\right), \quad v>4 .
$$

It follows, from Theorem 3.4 and (3.17)-(3.19), that

$$
r(t)=\sum_{k=0}^{\infty} C_{k}(v) e_{k}\left(\chi_{v}^{2}(t)\right), \quad C_{k}(v)=\frac{v}{2} \int_{0}^{\infty} \frac{p_{v / 2}(x) e_{k}(x) \mathrm{d} x}{x},
$$

and

$$
\sum_{k=0}^{\infty} C_{k}^{2}(v)=\frac{v^{2}}{4} \int_{0}^{\infty} \frac{p_{v / 2}(x) e_{k}(x) \mathrm{d} x}{x^{2}}<\infty, \quad v>4
$$

From (3.15), (3.16), and (3.20), we obtain the following properties of the inverse gamma process (3.18):

$$
\begin{aligned}
\mathrm{E}\{r(t)\} & =\frac{v}{v-2}, & v>2, \\
\operatorname{var}\{r(t)\} & =\frac{2 v^{2}}{(v-1)^{2}(v-2)}, & v>4,
\end{aligned}
$$


and

$$
\operatorname{cov}(r(t), r(t+\tau))=\sum_{k=1}^{\infty} C_{k}^{2}(v) \rho_{\eta}^{2 k}(\tau), \quad v>4 .
$$

Note that $\rho_{\eta}(\tau), \tau \in \mathbb{R}$, is a correlation function of the Gaussian process $\eta(t), t \in \mathbb{R}$, and takes the form

$$
\rho_{\eta}(\tau)=\frac{\operatorname{cov}(r(t), r(t+\tau))}{\operatorname{var}\{r(t)\}}=\frac{(v-1)^{2}(v-2)}{2 v^{2}} \sum_{k=1}^{\infty} C_{k}^{2}(v) \rho_{\eta}^{2 k}(\tau),
$$

where $v>4$ and $\tau \in \mathbb{R}$.

On the other hand, the bivariate PDF of a random vector $(r(t), r(t+\tau))$ is of the form

$$
\frac{v^{2}}{4 x^{2} y^{2}} p_{\nu / 2}\left(\frac{v}{2 x}, \frac{v}{2 y} ; \gamma\right), \quad x>0, y>0, \gamma=\rho_{\eta}^{2}(\tau)
$$

where $p_{v / 2}(u, w ; \gamma)$ is as defined in (3.14). If $v>4$, the correlation function $\rho_{\eta}(\tau)$ can be computed directly from the integral

$$
\rho_{\eta}(\tau)=\frac{1}{8}(v-1)^{2}(v-2) \int_{0}^{\infty} \int_{0}^{\infty}\left(x-\frac{v}{v-2}\right)\left(y-\frac{v}{v-2}\right) \frac{p_{v / 2}(v / 2 x, v / 2 y ; \gamma)}{x^{2} y^{2}} \mathrm{~d} x \mathrm{~d} y,
$$

where $p_{v / 2}(u, w ; \gamma)$ is as defined in (3.14) and $\gamma=\rho_{\eta}^{2}(\tau)$.

We can summarize our results in the following statement.

Theorem 3.5. For every integer $v \geq 1$, there exists a strictly stationary process (3.18) that has marginal inverse gamma distribution $\mathrm{R} \Gamma\left(\frac{1}{2} v, \frac{1}{2} v\right)$ with expectation (3.21), variance (3.22), and correlation function (3.23) or (3.24), when $v>4$.

A stochastic process (3.12) with $\nu \geq 2$ and $\mu=0$ can be considered to be a nonlinear transformation of the vector Gaussian process $\left(\eta_{0}(t), \eta_{1}(t), \ldots, \eta_{v}(t)\right) \in \mathbb{R}^{v+1}, t \in \mathbb{R}$; that is,

$$
X_{t}=F\left(\eta_{0}(t), \eta_{1}(t), \ldots, \eta_{\nu}(t)\right), \quad t \in \mathbb{R},
$$

with the nonlinear function

$$
\begin{gathered}
F\left(u_{0}, u_{1}, \ldots, u_{v}\right)=\frac{u_{0}}{\sqrt{\left(u_{1}^{2}+\cdots+u_{v}^{2}\right) / v}} \in L_{2}\left(\mathbb{R}^{v+1}, \phi(\boldsymbol{u}) \mathrm{d} \boldsymbol{u}\right), \\
\phi(\boldsymbol{u}) \equiv \phi\left(u_{0}, \ldots, u_{v}\right)=\varphi\left(u_{0}\right) \varphi\left(u_{1}\right) \cdots \varphi\left(u_{v}\right), \quad \varphi(s)=\frac{1}{\sqrt{2 \pi}} \mathrm{e}^{-s^{2} / 2}, \quad s \in \mathbb{R} .
\end{gathered}
$$

It is well known that the complete orthogonal system in the Hilbert space $L_{2}\left(\mathbb{R}^{v+1}, \phi(\boldsymbol{u}) \mathrm{d} \boldsymbol{u}\right)$ takes the form

$$
e_{k_{0}, k_{1}, \ldots, k_{v}}\left(u_{0}, \ldots, u_{v}\right)=\prod_{j=0}^{v} H_{k_{j}}\left(u_{j}\right)
$$

where $H_{v}(s), s \in \mathbb{R}, v=0,1,2, \ldots$, are Hermite polynomials (see, e.g. Courant and Hilbert (1953, Section II.9.4) or Leonenko (1999, pp. 170-174)).

In this Hilbert space, we have

$$
\frac{u_{0}}{\sqrt{\left(u_{1}^{2}+\cdots+u_{v}^{2}\right) / v}}=\sum_{m=0}^{\infty} \sum_{k_{0}+\cdots+k_{v}=m}^{\infty} \frac{C_{k_{0}, \ldots, k_{v}}}{k_{0} ! \cdots k_{\nu} !} e_{k_{0}, \ldots, k_{v}}\left(u_{0}, \ldots, u_{v}\right),
$$


where

$$
C_{k_{0}, \ldots, k_{v}}=\int_{\mathbb{R}^{v+1}} \frac{u_{0}}{\sqrt{\left(u_{1}^{2}+\cdots+u_{v}^{2}\right) / v}} e_{k_{0}, \ldots, k_{v}}\left(u_{0}, \ldots, u_{v}\right) \varphi\left(u_{0}, \ldots, u_{v}\right) \mathrm{d} u_{0} \cdots \mathrm{d} u_{v}
$$

and

$$
\sum_{m=0}^{\infty} \sum_{k_{0}+\cdots+k_{v}=m}^{\infty} \frac{C_{k_{0}, \ldots, k_{v}}^{2}}{k_{0} ! \cdots k_{\nu} !}=\int_{\mathbb{R}^{v+1}}\left(\frac{u_{0}}{\sqrt{\left(u_{1}^{2}+\cdots+u_{v}^{2}\right) / v}}\right)^{2} \varphi\left(u_{0}, \ldots, u_{v}\right) \mathrm{d} u_{0} \cdots \mathrm{d} u_{v} .
$$

Thus, in a Hilbert space of random variables with finite second moments, we obtain

$$
X_{t}=\sum_{m=0}^{\infty} \sum_{k_{0}+\cdots+k_{\nu}=m}^{\infty} \frac{C_{k_{0}, \ldots, k_{v}}}{k_{0} ! \cdots k_{\nu} !} e_{k_{0}, \ldots, k_{v}}\left(\eta_{0}(t), \ldots, \eta_{\nu}(t)\right)
$$

and, by using the orthogonality properties of Hermite polynomials, we have

$$
\mathrm{E}\left\{X_{t}\right\}=C_{0, \ldots, 0}=0
$$

and

$$
\operatorname{cov}\left(X_{t}, X_{t+\tau}\right)=\sum_{m=1}^{\infty} \rho_{\eta}^{m}(\tau) \sum_{k_{0}+\cdots+k_{v}=m}^{\infty} \frac{C_{k_{0}, \ldots, k_{\nu}}}{k_{0} ! \cdots k_{\nu} !} .
$$

If the correlation function $\rho_{\eta}(\tau)$ is of the form (3.11), we obtain a stationary Student process $X_{t}, t \in \mathbb{R}$, with long-range dependence.

\section{Associated self-similar processes}

In order to construct an associated self-similar stochastic process, we shall use the transformation of Lamperti (1962) and the results of Barndorff-Nielsen and Pérez-Abreu (1999).

Let $X_{t}, t \in \mathbb{R}$, be a strictly stationary process and define, for $0<H<1$,

$$
X_{t}^{*}=t^{H} X_{\log t}, \quad X_{0}^{*}=0, \quad t>0 .
$$

Then the process $X_{t}^{*}, t \geq 0$, is self-similar with exponent $H$, that is $X_{c t}^{*} \stackrel{\mathrm{D}}{=} c^{H} X_{t}^{*}$.

In this case, $X_{t}^{*}$ may or may not have strictly stationary increments. For instance, if we apply the Lamperti transformation (4.1) to the stationary Student process (3.4), or to the stationary Student process (3.7) with covariance function (3.11), we obtain self-similar processes that do not have strictly stationary increments.

If $X_{t}, t \in \mathbb{R}$, is a stationary Student process of type (3.4), then the covariance function of the Lamperti H-self-similar process (4.1) is of the form

$$
\operatorname{cov}\left(X_{t}^{*}, X_{s}^{*}\right)=\frac{\delta^{2} \Gamma\left(\frac{1}{2}(v-2)\right)}{2 \Gamma\left(\frac{1}{2} v\right)}(t s)^{H}\left(\frac{t}{s}\right)^{\lambda}, \quad \lambda>0, t<s,
$$

while, if $X_{t}, t \in \mathbb{R}$, is a stationary Student process of type (3.7), with covariance function (3.11), then the covariance function of the Lamperti Student H-self-similar process (4.1) takes the following form, where $0<H<1,0<\alpha \leq 1$, and $\varkappa \in \mathbb{R}$ :

$$
\operatorname{cov}\left(X_{t}^{*}, X_{s}^{*}\right)=(t s)^{H} \cos \left(\varkappa \log \left(\frac{s}{t}\right)\right)\left[1+\log ^{2}\left(\frac{s}{t}\right)\right]^{-\alpha / 2} .
$$


For both processes, we have $\mathrm{e}^{-H t} X_{\mathrm{e}^{t}}^{*} \sim T(\nu, \delta, \mu)$ and $X_{1}^{*}=X_{0}$, where $X_{0} \sim T(v, \delta, \mu)$. Note that (see Heyde and Gay (2002)) the covariance function of the self-similar process $X_{t}^{*}, t \geq 0$, with stationary second-order increments must be of the form

$$
\operatorname{cov}\left(X_{t}^{*}, X_{s}^{*}\right)=\frac{1}{2} \mathrm{E}\left\{X^{*}(1)\right\}^{2}\left\{s^{2 H}+t^{2 H}+|t-s|^{2 H}\right\}, \quad t, s \geq 0 .
$$

In this case, from Proposition 7.2.10 of Samorodnitsky and Taqqu (1994), we find that, for $j=0,1,2, \ldots$, the differenced sequence $Y_{j}=X_{j+1}^{*}-X_{j}^{*}$ is second-order stationary with covariance function

$$
\operatorname{cov}\left(Y_{0}, Y_{j}\right)=\frac{1}{2}\left[|j+1|^{2 H}-|j|^{2 H}+|j-1|^{2 H}\right] \sim H(2 H-1) j^{2 H-2}
$$

as $j \rightarrow \infty$, with $H \neq \frac{1}{2}$, meaning that $Y_{j}$ is a stationary process with long-range dependence when $H \in\left(\frac{1}{2}, 1\right)$ (see Heyde and Yang (1997)).

Equivalently, the correlation function of the associated stationary process in (4.1) must be of the form (see Theorems 2 and 3 of Barndorff-Nielsen and Pérez-Abreu (1999))

$$
\rho_{X}(\tau)=\cosh (H \tau)-2^{2 H-1} \sinh ^{2 H}\left(\frac{1}{2} \tau\right)=\int_{\mathbb{R}} \cos (\lambda \tau) F_{H}(\mathrm{~d} \lambda), \quad 0<H<1,
$$

where the spectral function $F_{H}$ has the spectral density

$$
f_{H}(\lambda)=\frac{1}{2 \pi} \sum_{j=0}^{\infty}(-1)^{j-1}\left(\begin{array}{c}
2 H \\
j
\end{array}\right)(j-H)\left\{(j-H)^{2}+\lambda^{2}\right\}^{-1}, \quad \lambda \in \mathbb{R}, 0<H<1 .
$$

Now, if we choose $\mu=0$ in (3.7) and let $F=F_{H}$ from (4.3), with spectral density (4.4), we obtain the $H$-self-similar process

$$
X_{t}^{*}=t^{H} \int_{\mathbb{R}} \cos (\lambda \log t) W_{1}\left\{R_{1}\left(F_{H}(\mathrm{~d} \lambda)\right)\right\}+t^{H} \int_{\mathbb{R}} \sin (\lambda \log t) W_{2}\left\{R_{2}\left(F_{H}(\mathrm{~d} \lambda)\right)\right\},
$$

where $t>0 ; X_{0}^{*}=0 ; W_{1}(t)$ and $W_{2}(t)$ are two independent standard Brownian motions; and $R_{1}(t)$ and $R_{2}(t)$ are two independent Lévy processes independent of $W_{1}(t)$ and $W_{2}(t)$ and such that $R_{i}(1) \sim \mathrm{R} \Gamma\left(\frac{1}{2} v, \frac{1}{2} \sigma^{2}\right), i=1,2, v>4$.

The process $\mathrm{e}^{-H t} X_{\mathrm{e}^{t}}^{*}, t>0$, is strictly stationary and has the Student distribution $T(v, \delta, 0)$. The associated differenced sequence $Y_{j}=X_{j+1}^{*}-X_{j}^{*}, j=1,2, \ldots$, is a second-order stationary process with covariance function (4.2). Note that

$$
X_{j}^{*} \stackrel{\mathrm{D}}{=} W_{1}\left(R_{1}(1)\right) \sim T(\nu, \delta, 0) \quad \text { and } \quad Y_{j} \sim T(v, \delta, 0), \quad j=0,1,2, \ldots
$$

\section{A risky asset model with strong dependence through fractal activity time}

Heyde (1999) (see also Heyde and Liu (2001), Heyde and Gay (2002), and Barndorff-Nielsen et al. (2002)) introduced a fractal activity time geometrical Brownian motion (FATGBM) as a model for risky assets. We are now going to study this model in more detail.

\subsection{Existence of FATGBM}

The paradigmatic model in mathematical finance is the geometric Brownian motion, otherwise known as the Black-Scholes model. In this model, the price $S_{t}$, at time $t$, of a risky asset is

$$
S_{t}=S_{0} \exp [\mu t+\sigma W(t)]
$$


where $\mu, \sigma^{2}>0$ are fixed constants and $W=\{W(t), t \geq 0\}$ is a standard Brownian motion. Note that (5.1) is the Itô solution to the SDE

$$
\mathrm{d} S(t)=S(t)\left(\left(\mu+\frac{1}{2} \sigma^{2}\right) \mathrm{d} t+\sigma \mathrm{d} W(t)\right) .
$$

Then, the one-period returns process is

$$
X_{t}=\log \left(\frac{S_{t}}{S_{t-1}}\right)=\mu+\sigma(W(t)-W(t-1)) .
$$

The following economically testable properties are consequences of the formulation (5.1), (5.2).

(i) The process $\left\{X_{t}\right\}$ is stationary Gaussian (so it is symmetric and has zero kurtosis).

(ii) $\left\{X_{t}\right\}$ has uncorrelated (and so independent) increments.

(iii) $\operatorname{cov}\left(\left(X_{t+k}-\mu\right)^{2},\left(X_{t}-\mu\right)^{2}\right)=0$, i.e. squared returns are independent.

(iv) $\operatorname{cov}\left(\left|X_{t+k}-\mu\right|,\left|X_{t}-\mu\right|\right)=0$, i.e. absolute returns are uncorrelated.

The Black-Scholes model plays a central role in financial theory. The associated arbitragefree asset pricing methodology has important applications and is widely used in practice.

Nonetheless, the original model has significant shortcomings. The following features of stock returns are well documented in the financial and econometric literature (see, for example, Heyde (1999), Heyde and Liu (2001), and references therein).

1. The returns process is uncorrelated.

2. Long-range dependence is present in the absolute and squared returns.

3. Returns have leptokurtic empirical distributions, i.e. higher-peaked and heavier-tailed than Gaussian distributions.

Heyde (1999) described a model similar to the Black-Scholes model for the stock price, which used geometric Brownian motion with fractal activity time (the FATGBM stock price model). The stock price is modelled as

$$
P_{t}=P_{0} \exp \left[\mu t+\sigma W\left(T_{t}\right)\right]
$$

Here, $\mu$ and $\sigma$ are constants and $W$ is a standard Brownian motion. However, the process $T=\left\{T_{t}, t \geq 0\right\}$, assumed to be independent of $W$, is neither clock nor intrinsic time, but rather an activity or market time that is not observed directly but which is strongly correlated with the trading volume of the stock. It is supposed that $T$ is a strictly increasing process with stationary differences and heavy-tailed finite-dimensional distributions.

There is strong empirical evidence in support of this model (see Heyde (1999), Heyde and Liu (2001), and Heyde and Gay (2002)), and that, to a good degree of first approximation, the process $T_{c t}-c t$ is asymptotically self-similar; that is,

$$
T_{c t}-c t \stackrel{\mathrm{D}}{\cong} c^{H}\left(T_{t}-t\right), \quad 0<H<1 .
$$

Exact self-similarity when $T$ is increasing is not possible, because, if (5.4) held exactly for all $t>0$ and $c>0$ then, for any $0<\Delta<1$,

$$
T_{t+\Delta}-T_{t}-\Delta \stackrel{\mathrm{D}}{=} T_{\Delta}-\Delta \stackrel{\mathrm{D}}{=} \Delta^{H}\left(T_{1}-1\right)
$$


and

$$
\mathrm{P}\left(T_{t+\Delta}-T_{t}<0\right)=\mathrm{P}\left(\Delta^{H} T_{1}<\Delta^{H}-\Delta\right)=\mathrm{P}\left(T_{1}<1-\Delta^{1-H}\right)>0 .
$$

Now the one-period stock returns process is

$$
X_{t}=\mu+\sigma\left(W\left(T_{t}\right)-W\left(T_{t-1}\right)\right) \stackrel{\mathrm{D}}{=} \mu+\sigma\left(T_{t}-T_{t-1}\right)^{1 / 2} W(1),
$$

using the scaling law of Brownian motion.

From (5.3)-(5.5), we obtain the following features of the FATGBM stock returns process $X=\left\{X_{t}\right\}$ (see Heyde (1999)).

(a) Stock returns are uncorrelated, i.e.

$$
\operatorname{cov}\left(X_{t+k}, X_{t}\right)=\sigma^{2} \mathrm{E}\left\{\left[W\left(T_{t+k}\right)-W\left(T_{t+k-1}\right)\right]\left[W\left(T_{t}\right)-W\left(T_{t-1}\right)\right]\right\}=0,
$$

since the Brownian motion has independent increments and $T$ is independent of $W$.

(b) There is long-range dependence in the absolute and squared returns: if $\mathrm{E}\left\{\tau_{t}^{2}\right\}<\infty$, where

$$
\tau_{t}=T_{t}-T_{t-1}
$$

then

$$
\operatorname{cov}\left(\left\{\left(X_{t}-\mu\right)^{2},\left(X_{t+k}-\mu\right)^{2}\right\}\right)=\sigma^{4} \operatorname{cov}\left(\tau_{t+k}, \tau_{t}\right),
$$

so if the $\left\{T_{t}\right\}$ process has long-range dependence of its increments $\left\{\tau_{t}\right\}$, then the same structure is present in the process $X_{t}^{2}$. Similarly,

$$
\operatorname{cov}\left(\left|X_{t}-\mu\right|,\left|X_{t+k}-\mu\right|\right)=\sigma^{2}(\mathrm{E}\{|W(1)|\})^{2} \operatorname{cov}\left(\tau_{t}^{1 / 2}, \tau_{t+k}^{1 / 2}\right), \quad \mathrm{E}\{|W(1)|\}=2 / \pi .
$$

(c) There are leptokurtic distributions of $X_{t}$; in particular,

$$
\operatorname{kurtosis}\left(X_{t}\right)=3\left(1+\operatorname{var} \tau_{t}\right)>3 .
$$

We now propose some models with properties (5.3)-(5.8), in which the process $X_{t}$ has both the above properties and the symmetric scaled $t$-distribution.

Suppose that we are given a Student distribution $T\left(v, v^{1 / 2}, \mu\right)$ with the PDF (2.1), and we wish to construct a stochastic process $T=\left\{T_{t}, t \geq 0\right\}-$ assumed to be independent of the standard Brownian motion $W(t)$ - such that properties (5.5), (5.6), and (5.8) hold, the oneperiod stock returns process $X_{t}$ has distribution $T\left(\nu, v^{1 / 2}, \mu\right)$, and $\left\{X_{t}\right\}$ displays long-range dependence.

Let us consider the strictly stationary process similar to (3.18)-(3.20), i.e. $r(t)=G\left(\chi_{v}^{2}(t)\right)$, but now with $G(u)=\left(\frac{1}{2} v-1\right) / u$, marginal $\left[\left(\frac{1}{2} v-1\right) / \frac{1}{2} v\right] \mathrm{R} \Gamma\left(\frac{1}{2} v, \frac{1}{2} v\right)$ distribution, and longrange dependence. If $v>4$ then

$$
C_{0}(v)=\left(\frac{1}{2} v-1\right) \int_{0}^{\infty} p_{v / 2}(x) \frac{\mathrm{d} x}{x}=1, \quad C_{1}(v)=\left(\frac{1}{2} v-1\right) \int_{0}^{\infty} p_{v / 2}(x) e_{1}(x) \frac{\mathrm{d} x}{x} \neq 0,
$$

where the first generalized Laguerre polynomial is given explicitly by

$$
e_{1}(u)=\sqrt{\frac{2}{v}}\left(\frac{1}{2} v-u\right)
$$


and, hence,

$$
e_{1}\left(\chi_{v}^{2}(t)\right)=-\frac{1}{\sqrt{2 v}} \sum_{j=1}^{v}\left(\eta_{j}^{2}(t)-1\right)
$$

where $\eta_{1}(t), \ldots, \eta_{v}(t)$ are independent copies of the stationary Gaussian process $\eta(t)$ with zero mean and covariance function $\rho_{\eta}(\tau)=\left(1+\tau^{2}\right)^{-\alpha / 2}, 0<\alpha<\frac{1}{2}$.

From Taqqu (1975) (see also Berman (1992) and Leonenko (1999)), we obtain

$$
\lim _{n \rightarrow \infty} \operatorname{var}\left\{\frac{1}{n^{1-\alpha}}\left[\sum_{s=1}^{\lfloor n t\rfloor} G\left(\chi_{v}^{2}(s)\right)-\lfloor n t\rfloor\right]-\frac{C_{1}(v)}{n^{1-\alpha}} \sum_{s=1}^{\lfloor n t\rfloor} e_{1}\left(\chi_{v}^{2}(s)\right)\right\}=0, \quad t \geq 0,
$$

where

$$
G(u)=\left(\frac{1}{2} v-1\right) u \in L_{2}\left((0, \infty), p_{v / 2}(x) \mathrm{d} x\right)
$$

if $v>4$, and $\lfloor\cdot\rfloor$ denotes the largest integer less than or equal to its argument. Also, from the same reference,

$$
\frac{C_{1}(v)}{n^{1-\alpha}} \sum_{s=1}^{\lfloor n t\rfloor} e_{1}\left(\chi_{v}^{2}(s)\right)=-\frac{C_{1}(v)}{\sqrt{2 v} n^{1-\alpha}} \sum_{j=1}^{v} \frac{1}{n^{1-\alpha}} \sum_{s=1}^{\lfloor n t\rfloor}\left(\eta_{j}^{2}(s)-1\right), \quad t \geq 0,
$$

converges, as $n \rightarrow \infty$, to the stochastic process

$$
R_{\infty}(t)=-\frac{C_{1}(v)}{\sqrt{2 v}} \sum_{j=1}^{v} R_{j}(t)
$$

in the sense of finite-dimensional distributions. Here, $R_{1}(t), \ldots, R_{v}(t)$ are independent copies of the Rosenblatt process $R(t), t \geq 0$, with characteristic function (see Taqqu (1975)) of the form

$$
\begin{aligned}
\Phi\left(z_{1}, \ldots, z_{q}\right) & =\mathrm{E}\left\{\exp \left[\mathrm{i} \sum_{p=1}^{q} z_{p} R\left(t_{p}\right)\right]\right\} \\
& =\exp \left[\frac{1}{2} \sum_{k=2}^{\infty} \frac{(2 \mathrm{i})^{k}}{k} \sum_{s_{1}, \ldots, s_{k} \in\{1,2, \ldots, q\}} z_{s_{1}} z_{s_{2}} \cdots z_{s_{q}} s_{\alpha}(k)\right],
\end{aligned}
$$

where

$$
s_{\alpha}(k)=\int_{0}^{t_{s_{1}}} \cdots \int_{0}^{t_{s_{q}}} \frac{\mathrm{d} x_{1} \cdots \mathrm{d} x_{k}}{\left|x_{1}-x_{2}\right|^{\alpha}\left|x_{2}-x_{3}\right|^{\alpha} \cdots\left|x_{k}-x_{1}\right|^{\alpha}}, \quad 0<\alpha<\frac{1}{2} .
$$

The stochastic process (5.9) is self-similar with parameter $H=1-\alpha \in\left(\frac{1}{2}, 1\right)$, i.e.

$$
R(c t) \stackrel{\mathrm{D}}{=} c^{H} R(t), \quad t \geq 0, c>0 .
$$

This indicates that the process

$$
T_{\lfloor n t\rfloor}=\sum_{s=1}^{\lfloor n t\rfloor} \frac{\left(\frac{1}{2} v-1\right)}{\frac{1}{2}\left(\eta_{1}^{2}(s)+\cdots+\eta_{v}^{2}(s)\right)}-\lfloor n t\rfloor
$$


has the asymptotic self-similar property (5.4), with parameter $H=1-\alpha \in\left(\frac{1}{2}, 1\right)$. That is, to a good degree of first approximation, the process $T_{c t}-c t, c>0$, can be approximated, in the sense of finite-dimensional distributions, by the process $c^{H}\left(T_{t}-t\right)$ and, thus, the distribution of the random variables $T_{t}$ can be approximated by the distribution of the random variables $t+t^{H}\left(T_{1}-1\right)$, where $T_{1} \sim\left[\left(\frac{1}{2} v-1\right) / \frac{1}{2} v\right] \mathrm{R} \Gamma\left(\frac{1}{2} v, \frac{1}{2} v\right)$. The process (5.10) exhibits long-range dependence. The existence of a FATGBM model follows.

\subsection{Pricing formulae}

The self-similarity relationship (5.4), being empirically based, provides a key to the pricing of derivatives for the FATGBM model, which is similarly based. This is in contrast to the usual stochastic volatility models, in which the volatility SDE has no such foundation.

A pricing formula for European call and put options was proposed by Heyde and Gay (2002). A sketch of the reasoning follows. Suppose that, in a certain market, there is a deterministic money market account $\beta_{t}=\beta_{0} \mathrm{e}^{r t}$ and a stock price $S_{t}$. In the no-arbitrage pricing regime, the discounted stock price $\left\{S_{t} / \beta_{t}\right\}$ under $\mathrm{Q}$, a martingale measure equivalent to that induced by $\left\{S_{t}\right\}$, is a martingale $M_{t}$, say. Then, under $\mathrm{Q}$ we must have

$$
\mathrm{d} M_{t}=M_{t}\left\{(\mu-r) \mathrm{d} t+\sigma \mathrm{d} W\left(T_{t}\right)\right\}
$$

Since such processes can only be martingales if $\mu=r$, we need to choose $\mu=r$ to price any option of this stock price. Hence, the call expiring at time $t$ is priced in the present by using the pricing equation

$$
S_{t}^{*}=S_{0} \exp \left[\left(r t-\frac{1}{2} \sigma^{2} T_{t}\right)+\sigma W\left(T_{t}\right)\right]
$$

and the measure $\mathrm{Q}$.

Denote the call price by $C$. Then, as with the Black-Scholes formula,

$$
C=\mathrm{E}_{\mathrm{Q}}\left\{\mathrm{e}^{-r t}\left(S_{t}^{*}-K\right)^{+}\right\}=\mathrm{E}_{\mathrm{Q}}\left\{\mathrm{E}_{\mathrm{Q}}\left\{\mathrm{e}^{-r t}\left(S_{t}^{*}-K\right)^{+} \mid\left\{T_{t}\right\}\right\}\right\}=\mathrm{E}_{\mathrm{Q}}\left\{P_{t}\right\}=\mathrm{E}\left\{P_{t}\right\},
$$

where

$$
P_{t}=S_{0} \Phi\left[\frac{\log \left[S_{0} / K\right]+r t+\frac{1}{2} \sigma^{2} T_{t}}{\sigma \sqrt{T_{t}}}\right]-K \mathrm{e}^{-r t} \Phi\left[\frac{\log \left[S_{0} / K\right]+r t-\frac{1}{2} \sigma^{2} T_{t}}{\sigma \sqrt{T_{t}}}\right] .
$$

Here $\Phi$ is the distribution function of a standard normal distribution and the expectation of $P_{t}$ is the same under the real-world measure as it is under the nonunique Q. Note that this is an incomplete market situation but that the price calculated above is natural in the sense of being a conditional Black-Scholes price. Indeed, $P_{t}$ is the Black-Scholes price conditional on $T_{t}$, and $C$ is calculated by taking the expectation of $P_{t}$ with respect to the real-world measure.

For pricing, $T_{t}$ can be chosen to have the distribution $t+t^{H}\left(T_{1}-1\right)$, with

$$
T_{1} \sim \mathrm{R} \Gamma\left(\frac{1}{2} v, \frac{1}{2}(v-2)\right),
$$

and $W\left(T_{1}\right)$ has the distribution $((v-2) / v)^{1 / 2} T\left(v, v^{1 / 2}, 0\right), v>4,0<H<1$.

The put option price comes from the call-put parity relation:

$$
\text { put price }=\text { call price }- \text { stock price }+ \text { present value of exercise price. }
$$

See Heyde and Gay (2002) for more details and some examples. 


\section{Appendix A. Modified Bessel functions of the third kind}

In this appendix, a number of results concerning the modified Bessel functions of the third kind are collected (see Watson (1958) or Kotz et al. (2001, pp. 314-317)).

The modified Bessel function of the third kind, with index $\lambda \in \mathbb{R}$, can be defined by the integral representation

$$
K_{\lambda}(x)=\frac{1}{2} \int_{0}^{\infty} u^{\lambda-1} \exp \left[\frac{1}{2} x\left(u+\frac{1}{u}\right)\right] \mathrm{d} u, \quad x>0 .
$$

The function $K_{\lambda}(x)$ is a continuous, positive function of $\lambda \geq 0$ and $x>0$. If $\lambda \geq 0$ is fixed then, throughout the $x$ interval $(0, \infty)$, the function $K_{\lambda}(x)$ is positive and decreasing.

If $\lambda$ is fixed then, as $x \rightarrow 0+$,

$$
K_{\lambda}(x) \sim \Gamma(\lambda) 2^{\lambda-1} x^{-\lambda}, \quad \lambda>0, \quad K_{0}(x) \sim \log (1 / x) .
$$
form

For $\lambda=r+\frac{1}{2}$, where $r$ is a nonnegative integer, the Bessel function $K_{\lambda}(x)$ has the closed

$$
K_{r+1 / 2}(x)=\sqrt{\frac{\pi}{2 x}} \mathrm{e}^{-x} \sum_{k=0}^{r} \frac{(r+k) !}{(r-k) ! k !}(2 x)^{-k} .
$$

\section{Acknowledgements}

The authors gratefully acknowledge financial support for this work from the Australian Research Council (grant DP 0345577) and the EPSRC (grant RCMT091).

\section{References}

Ait-Sahalia, Y. (1996). Nonparametric pricing of interest rate derivative securities. Econometrica 64, 527-560.

Anh, V. V., Heyde, C. C. And Leonenko, N. N. (2002). Dynamic models of long-memory processes driven by Lévy noise. J. Appl. Prob. 39, 730-747.

Anh, V. V., Knopova, V. P. And Leonenko, N. N. (2004). Continuous-time stochastic processes with cyclical longrange dependence. Austral. N. Z. J. Statist. 46, 275-296.

BARNDORFF-NiElSEN, O. E. (1977). Exponentially decreasing distributions for logarithm of particle size. Proc. R. Soc. London A 353, 401-419.

Barndorff-Nielsen, O. E. (1978). Hyperbolic distributions and distributions on hyperbolae. Scand. J. Statist. 5, $151-157$.

BARndORfF-Nielsen, O. E. (1998). Processes of normal inverse Gaussian type. Finance Stoch. 2, 41-68.

Barndorff-Nielsen, O. E. (2001). Superposition of Ornstein-Uhlenbeck type processes. Theory Prob. Appl. 45, 175-194.

BARNDORFF-Nielsen, O. E. AND HALGREEN, C. (1977). Infinite divisibility of the hyperbolic and generalized inverse Gaussian distributions. Z. Wahrscheinlichkeitsth. 38, 309-312.

BARNDORFF-Nielsen, O. E. ANd LeONENKo, N. N. (2005). Spectral properties of superpositions of Ornstein-Uhlenbeck type processes. To appear in Method. Comput. Appl. Prob.

Barndorff-Nielsen, O. E. ANd PÉrez-Abreu, V. (1999). Stationary and self-similar processes driven by Lévy processes. Stoch. Process. Appl. 84, 357-369.

BarndorfF-Nielsen, O. E. AND Shephard, N. (2001). Non-Gaussian Ornstein-Uhlenbeck-based models and some of their uses in financial econometrics. J. R. Statist. Soc. B 63, 167-241.

Barndorff-Nielsen, O. E., Jensen, J. L. And Sørensen, M. (1998). Some stationary processes in discrete and continuous time. Adv. Appl. Prob. 30, 989-1007.

Barndorff-Nielsen, O. E., Nicalato, E. and Shephard, N. (2002). Some recent developments in stochastic volatility modelling. Quant. Finance 2, 11-23.

Bateman, H. And Erdélyi, A. (1953). Higher Transcendental Functions, Vol. 2. McGraw-Hill, New York.

Berman, S. M. (1992). Sojourns and Extremes of Stochastic Processes. Wadsworth and Brooks, Pacific Grove, CA.

Bertoin, J. (1996). Lévy Processes (Camb. Tracts Math. 121). Cambridge University Press. 
Bibby, B. M. And Sørensen, M. (1997). A hyperbolic diffusion model for stock prices. Finance Stoch. 1, $25-41$.

Bibby, B. M., Skovgaard, I. M. And Sørensen, M. (2003). Diffusion-type models with given marginal and autocorrelation function. Bernoulli 11, 191-220.

Bingham, N. H. AND KIESEL, R. (2002). Semi-parametric modelling in finance: theoretical foundations. Quant. Finance 2, 241-250.

Bingham, N. H., Goldie, C. M. And Teugels, T. L. (1987). Regular Variation. Cambridge University Press.

Borland, L. (2002). A theory of non-Gaussian option pricing. Quant. Finance 2, 415-431.

Cont, R. And Tankov, P. (2004). Financial Modelling with Jump Processes. Chapman and Hall, Boca Raton, FL.

Costa, J., Hero, A. And Vignat, C. (2003). On solutions to multivariate maximum $\alpha$-entropy problems. In Proc. Energy Minimization Methods in Computer Vision and Pattern Recognition (Lisbon, July 2003; Lecture Notes Comput. Sci. 2683), Springer, Berlin, pp. 211-228.

Courant, R. And Hilbert, D. (1953). Methods of Mathematical Physics. Interscience, New York.

Cramér, A. J. And Leadbetter, M. R. (1967). Stationary and Related Stochastic Processes. John Wiley, New York.

Dreier, I. And Kotz, S. (2002). A note on the characteristic function of the $t$-distribution. Statist. Prob. Lett. 57, 221-224.

Eberlein, E. And Keller, U. (1995). Hyperbolic distributions in finance. Bernoulli 1, 281-299.

Grosswald, E. (1976). The Student $t$-distribution of any degree of freedom is infinitely divisible. Z. Wahrscheinlichkeitsth. 36, 103-109.

Halgreen, C. (1979). Self-decomposability of generalized inverse Gaussian and hyperbolic distributions. Z. Wahrscheinlichkeitsth. 47, 13-17.

Havdra, M. ANd Charvát, F. (1967). Quantification method of classification processes: concept of structural $\alpha$ entropy. Kybernetika 3, 30-35.

Heyde, C. C. (1999). A risky asset model with strong dependence through fractal activity time. J. Appl. Prob. 36, 1234-1239.

Heyde, C. C. And Gay, R. (2002). Fractals and contingent claims. Preprint, Australian National University.

Heyde, C. C. AND LiU, S. (2001). Empirical realities for a minimal description risky asset model. The need for fractal features. J. Korean Math. Soc. 38, 1047-1059.

Heyde, C. C. And Yang, Y. (1997). On defining long-range dependence. J. Appl. Prob. 34, 939-944.

Hurst, S. R. And Platen, E. (1997). The marginal distribution of returns and volatility. In $L_{1}$-Statistical Procedures and Related Topics (IMS Lecture Notes Monogr. Ser. 31), ed. Y. Dodge, IMS, Hayward, CA, pp. 301-314.

Hurst, S. R., Platen, E. And Rachev, S. R. (1997). Subordinated Markov models: a comparison. Finan. Eng. Japanese Markets 4, 97-124.

JuReK, Z. J. (2001). Remarks on the self-decomposability and new examples. Demonstr. Math. 34, 241-250.

Jurek, Z. J. And Mason, J. D. (1993). Operator-Limit Distributions in Probability Theory. John Wiley, New York.

Kotz, S., Kozubowski, T. J. And Podgorski, K. (2001). The Laplace Distribution and Generalizations. Birkhäuser, Boston, MA.

KWAPIEN, S. AND WoyCZYŃSKI, W. A. (1992). Random Series and Stochastic Integrals: Single and Multiple. Birkhäuser, Boston, MA.

Lamperti, J. W. (1962). Semi-stable stochastic process. Trans. Amer. Math. Soc. 104, 62-78.

LeOnenko, N. N. (1999). Limit Theorems for Random Fields with Singular Spectrum. Kluwer, Dordrecht.

Madan, D. B. And Seneta, E. (1990). The variance gamma (V.G.) model for share market returns. J. Business 63, 511-524.

Mandelbrot, B. B. (2001a). Scaling in financial prices. I. Tails and dependence. Quant. Finance 1, 113-123.

Mandelbrot, B. B. (2001b). Scaling in financial prices. II. Multifractals and the star equation. Quant. Finance 1, 124-130.

RaJPut, B. AND Rosiński, J. (1989). Spectral representations of infinitely divisible processes. Prob. Theory Relat. Fields 82, 451-487.

Rényi, A. (1961). On measures of entropy and application. In Proc. 4th Berkeley Symp. Math. Statist. Prob., Vol. 1, University of California Press, Berkeley, CA, pp. 547-561.

RosińsKi, J. (1991). On a class of infinitely divisible processes represented as mixtures of Gaussian processes. In Stable Processes and Related Topics, eds S. Cambanis, G. Samorodnitsky and M. S. Taqqu, Birkhäuser, Basel, pp. $27-41$.

Samorodnitsky, G. AND TaqQu, M. S. (1994). Stable Non-Gaussian Random Processes. Chapman and Hall, New York.

Sato, K. (1999). Lévy Processes and Infinitely Divisible Distributions. Cambridge University Press.

Schoutens, W. (2003). Lévy Processes in Finance: Pricing Financial Derivatives. John Wiley, Chichester.

Seneta, E. (2004). Fitting the variance-gamma model to financial data. In Stochastix Methods and Their Applications (J. Appl. Prob. Spec. Vol. 41A), Applied Probability Trust, Sheffield, pp. 177-187.

Sørensen, M. ANd Bibby, M. (2003). Hyperbolic processes in finance. In Handbook of Heavy Tailed Distributions in Finance, ed. S. T. Rachev, Elsevier, Amsterdam, pp. 211-248. 
TAQQU, M. S. (1975). Weak convergence to fractional Brownian motion and to the Rosenblatt process. Z. Wahrscheinlichkeitsth. 31, 287-302.

TARAmi, B. AND Pourahmadi, M. (2003). Multi-variate $t$ autoregressions: innovations, prediction variances and exact likelihood equations. J. Time Ser. Anal. 24, 739-754.

Tsallis, C. And Bukman, D. J. (1996). Anomalous diffusion in the presence of external forces: exact time-dependent solutions and their thermostatistical basis. Phys. Rev. E 54, 2197-2200.

Tsallis, C., Levy, S. V. F., Souza, A. M. C. and Maynard, R. (1995). Statistical-mechanical foundation of the ubiquity of Lévy distributions in nature. Phys. Rev. Lett. 75, 3589-3593.

Vignat, C. and Bercher, J. F. (2003). Analysis of signals in the Fisher-Shannon information plane. Phys. Rev. A 312, 27-33.

Watson, G. N. (1958). A Treatise on the Theory of Bessel Functions, 2nd edn. Cambridge University Press.

WitKovsky, V. (2002). Exact distribution of positive linear combinations of inverted chi-square random variables with odd degrees of freedom. Statist. Prob. Lett. 56, 45-50.

WoyCZyŃsKI, W. A. (1998). Burgers-KPZ Turbulence (Lecture Notes Math. 1700). Springer, Berlin. 